\title{
Perinuclear antineutrophil cytoplasmic autoantibodies and anti-Saccharomyces cerevisiae antibodies as serological markers are not specific in the identification of Crohn's disease and ulcerative colitis
}

\author{
Vishal Anand MD ${ }^{1}$, Anthony S Russell MD², Ross Tsuyuki PharmD MSc ${ }^{3}$, Richard Fedorak MD ${ }^{1}$
}

\begin{abstract}
V Anand, AS Russell, R Tsuyuki, R Fedorak. Perinuclear antineutrophil cytoplasmic autoantibodies and antiSaccharomyces cerevisiae antibodies as serological markers are not specific in the identification of Crohn's disease and ulcerative colitis. Can J Gastroenterol 2008;22(1):33-36.
\end{abstract}

OBJECTIVE: To evaluate the diagnostic accuracy of perinuclear antineutrophil cytoplasmic autoantibodies (pANCAs) and antiSaccharomyces cerevisiae antibodies (ASCAs), as single agents and in combination, for the diagnosis of Crohn's disease (CD) and ulcerative colitis (UC), including in cases of indeterminate colitis (IC). METHODS: The sera from a total of 98 patients were studied: 77 with CD, 16 with UC and five with IC. The medical records of these patients were reviewed for disease diagnosis, demographic data, and patient symptoms and medications. ELISAs were utilized to detect the presence of ASCAs and deoxyribonuclease-sensitive pANCAs, and these results were then compared with the patients' clinical data.

RESULTS: For UC, a positive pANCA test alone provided a sensitivity of $50 \%$ and a specificity of $82 \%$. For CD, a positive ASCA test alone provided a sensitivity of $40 \%$ and a specificity of $100 \%$. A combination of pANCA-positive and ASCA-negative results showed a sensitivity of $50 \%$ and specificity of $90 \%$ for the diagnosis of UC. Similarly, the combination of ASCA-positive and pANCA-negative results provided a sensitivity and specificity of $32 \%$ and $100 \%$ for the diagnosis of $\mathrm{CD}$, respectively. Interestingly, $80 \%$ of IC patients showed serology results consistent with UC.

CONCLUSIONS: Although this combination of serological markers provides a diagnostic tool with generally high specificities, the low sensitivities of these serological markers, most notably in terms of $\mathrm{CD}$, preclude the possibility that they can replace the tools currently used for inflammatory bowel disease diagnosis and management. It is possible, however, that these serological markers may prove beneficial in the management of IC.

Key Words: ANCA; Antineutrophil cytoplasmic antibody; AntiSaccharomyces cerevisiae antibody; ASCA; Crohn's disease; ELISA; Ulcerative colitis

Inflammatory bowel disease (IBD) is an umbrella term usually used to cover two idiopathic diseases: Crohn's disease (CD) and ulcerative colitis (UC). Although UC is more typically a mucosal inflammation of the large intestine (with extension to the rectum) and $\mathrm{CD}$ is typically a patchy transmural

\section{Anticorps cytoplasmiques antineutrophiles périnucléaires et anticorps anti-Saccharomyces cerevisia comme marqueurs sérologiques, non spécifiques dans l'identification de la maladie de Crohn et de la colite ulcéreuse}

\begin{abstract}
OBJECTIF : Évaluer la précision diagnostique des anticorps cytoplasmiques antineutrophiles périnucléaires (ou pANCA, pour perinuclear antineutrophil cytoplasmic autoantibodies) et des anticorps antiSaccharomyces cerevisiæ (ou ASCA, pour anti-Saccharomyces cerevisiæ antibodies) à titre de techniques utilisées seules ou en concomitance pour le diagnostic de la maladie de Crohn (MC) et de la colite ulcéreuse (CU), y compris dans les cas de colite indéterminée $(\mathrm{CI})$.

MÉTHODES : Des échantillons sériques de 98 patients en tout ont été analysés : 77 porteurs de $\mathrm{MC}, 16$, de CU et 5, de CI. Les dossiers médicaux de ces patients ont été passés en revue pour ce qui était de leurs diagnostics, données démographiques, symptômes et pharmacothérapies. La méthode ELISA a été utilisée pour déceler la présence des ASCA et des pANCA sensibles à la désoxyribonucléase et ces résultats ont ensuite été comparés aux données cliniques des patients.

RÉSULTATS : Pour la CU, un test pANCA-positif seul s'est accompagné d'une sensibilité de $50 \%$ et d'une spécificité de $82 \%$. Pour la MC, un test ASCA-positif seul a donné lieu à une sensibilité de $40 \%$ et à une spécificité de $100 \%$. En associant les résultats pANCA-positifs et ASCAnégatifs, on a obtenu une sensibilité de $50 \%$ et une spécificité de $90 \%$ pour le diagnostic de la CU. De même, en alliant les résultats ASCApositifs et pANCA-négatifs, on a obtenu une sensibilité et une spécificité de $32 \%$ et de $100 \%$, respectivement, pour le diagnostic de MC. Fait à noter, $80 \%$ des patients atteints de $\mathrm{CI}$ ont obtenu des résultats sérologiques concordant avec une CU.

CONCLUSIONS : Bien que cette association de marqueurs sérologiques constitue un outil diagnostique généralement doté de spécificités élevées, leurs faibles sensibilités, plus précisément vis-à-vis de la MC, les empêchent de remplacer les outils actuellement utilisés pour le diagnostic et la prise en charge des maladies inflammatoires de l'intestin. Il est possible, par contre, que ces marqueurs sérologiques se révèlent bénéfiques pour la prise en charge de la CI.
\end{abstract}

inflammation affecting any part of the intestinal tract, the two diseases often display overlapping symptoms and clinical presentations.

The current standard diagnostic process for IBD involves a combination of a thorough history, a complete physical

${ }^{1}$ Division of Gastroenterology; ${ }^{2}$ Division of Rheumatology; ${ }^{3}$ Epidemiology Coordinating and Research Centre, Faculty of Medicine and Dentistry, University of Alberta, Edmonton, Alberta

Correspondence: Dr Richard N Fedorak, Director, Centre of Excellence for Gastrointestinal Inflammation and Immunity Research, Division of Gastroenterology, University of Alberta, Zeidler Ledcor Building, Edmonton, Alberta T6G 2X8. Telephone 780-492-6941,

fax 780-492-8121, e-mail Richard.Fedorak@ualberta.ca

Received for publication November 11, 2006. Accepted March 27, 2007 
TABLE 1

Test results for determining ulcerative colitis in 93 inflammatory bowel disease patients

\begin{tabular}{lccccccc}
\hline & \multicolumn{2}{c}{$\begin{array}{c}\text { Crohn's } \\
\text { disease }\end{array}$} & $\begin{array}{c}\text { Ulcerative } \\
\text { colitis }\end{array}$ & & & & \\
Test result & $(\mathbf{n = 7 7 )}, \mathbf{n}$ & $(\mathbf{n = 1 6 )}, \mathbf{n}$ & Sensitivity & Specificity & PPV & NPV \\
\hline pANCA+ & 14 & 8 & 0.50 & 0.82 & 0.36 & 0.89 \\
pANCA+ and ASCA- & 8 & 8 & 0.50 & 0.90 & 0.50 & 0.90
\end{tabular}

ASCA- Anti-Saccharomyces cerevisiae antibody-negative; NPV Negative predictive value; $p A N C A+$ Perinuclear antineutrophil cytoplasmic autoantibodypositive; PPV Positive predictive value

examination, and a multitude of physiological investigations, including endoscopy, histology of any endoscopically obtained tissue specimens and diagnostic radiography. In the past several years, however, much attention has been paid to the possible clinical significance of serum markers, which may be useful in the diagnosis and management of IBD, most notably perinuclear antineutrophil cytoplasmic autoantibodies (pANCAs) and anti-Saccharomyces cerevisiae antibodies (ASCAs).

Since 1990, we have seen the emergence of studies on pANCAs and their association with UC. Most literature reports that between $60 \%$ and $80 \%$ of adults and children with UC test positive for serum pANCAs $(1,2)$. The pANCA is an autoantibody made by the lamina propria and mesenteric node lymphocytes, and although its exact epitope remains unknown, its antigen is suspected to be located on the inner side of the nuclear periphery and to be deoxyribonucleasesensitive (2).

Since 1988, ASCAs have also been gaining recognition in terms of their association with CD. An antibody that targets the phosphopeptidomannan part of the cell wall of $S$ cerevisiae (ie, Baker's yeast), ASCAs have consistently been found in $50 \%$ to $80 \%$ of CD patients (3).

Because the number of studies evaluating these serological markers is limited, especially those studies that consider these markers' combined applicability, we evaluated their precision as diagnostic tools for subsets of our IBD patient population. Specifically, we researched the sensitivity, specificity, positive predictive value (PPV) and negative predictive value (NPV) of pANCAs and ASCAs, used alone and in combination, for the diagnosis of both CD and UC.

\section{Patient population}

\section{PATIENTS AND METHODS}

A total of 98 patients enrolled in the present study between December 2004 and May 2005. Serum samples were collected from all participants. No specific inclusion or exclusion criteria were used in the identification of prospective participants. After each patient's serum was tested, all medical records of that patient were reviewed for data regarding the final disease diagnosis, basic demographic data, and patient symptoms and medications. In all cases, disease diagnosis was confirmed using endoscopic and histological criteria.

\section{ASCAs}

The ASCA kits were obtained from Euroimmun AG, Germany. The traditional indirect immunofluorescent assay method, in which slides with biochips of $S$ cerevisiae are incubated with either a $1: 100$ or $1: 1000$ dilution of patient serum for $30 \mathrm{~min}$ at room temperature, was followed. Different serum dilutions were used to correspond with either immunoglobulin
TABLE 2

Test results for determining Crohn's disease in 93 inflammatory bowel disease patients

\begin{tabular}{|c|c|c|c|c|c|c|}
\hline Test result & $\begin{array}{l}\text { Crohn's } \\
\text { disease } \\
(n=77), n\end{array}$ & $\begin{array}{l}\text { Ulcerative } \\
\text { colitis } \\
(n=16), n\end{array}$ & Sensitivity & Specificity & PPV & NPV \\
\hline ASCA+ & 31 & 0 & 0.40 & 1.00 & 1.00 & 0.26 \\
\hline pANCA - and ASCA & $A+25$ & 0 & 0.32 & 1.00 & 1.00 & 0.24 \\
\hline
\end{tabular}

ASCA+Anti-Saccharomyces cerevisiae antibody-positive; NPV Negative predictive value; pANCA- Perinuclear antineutrophil cytoplasmic autoantibodynegative; PPV Positive predictive value

(Ig) A-specific or IgG-specific fluorescein conjugates, as per kit directions. After a phosphate-buffered saline (PBS) wash was used to remove excess serum, the specific IgA or IgG conjugates were placed in appropriate wells and further incubated for $30 \mathrm{~min}$ at room temperature. After an additional wash with PBS, the slides were covered with a slip and read on a fluorescent microscope that detected whether the antibodies (which had been fluorescently labelled) were present. A positive reaction for either IgA or IgG was indicated by apple-green fluorescent staining of the fungi in question.

\section{pANCAs}

pANCA testing was performed on lab-prepared, ethanol-fixed neutrophil slides. Slides were prepared as per the protocol from the First International Workshop on ANCA (1988). Patient serum was diluted to a 1:20 concentration, then placed on cell spots and incubated for $20 \mathrm{~min}$ at room temperature. After a PBS wash and IgG-specific fluorescein, goat antihuman conjugate was placed on the spot and the whole sample was incubated at room temperature for an additional $20 \mathrm{~min}$. Slides were again covered with a slip and read on a fluorescent microscope, at which time the ANCA pattern was determined. The pattern of specific interest for correlation with the ASCA pattern was the pANCA, which exhibits fluorescence on the periphery of the nucleus, usually with no fluorescence in the cytoplasm.

\section{Statistical analysis}

The sensitivity, specificity, PPV and NPV were calculated for pANCA, ASCA and their combination for each diagnostic category.

\section{RESULTS}

Of the 98 patients included in the present study, 77 had CD, 16 had UC and five had indeterminate colitis (IC). The results of the study as they relate to these specific disease groups are summarized in Tables 1 and 2, as well as in Figure 1.

For the UC group, the pANCA test was found to be positive in eight of the 16 known UC patients. Thus, the pANCA test had a sensitivity and specificity of $50 \%$ and $82 \%$, respectively. In other words, the pANCA test, when used on its own, had a PPV of $36 \%$ and a NPV of $89 \%$ for UC.

Of the patients with CD $(n=77), 31$ were ASCA-positive $(+)$ and 46 were ASCA-negative (-). Of the $31 \mathrm{CD}$ patients who were ASCA+, 13 expressed both $\operatorname{IgA}$ and $\operatorname{IgG}$, 16 expressed only IgA and two expressed only IgG. Therefore, when examined on its own, the ASCA test for CD had a sensitivity and specificity of $40 \%$ and $100 \%$, respectively, providing a PPV of $100 \%$ and a NPV of $26 \%$. Remarkably, none of the UC patients tested positive for ASCA IgA or ASCA IgG. 
When the data from both assays were combined to show UC patients who were pANCA + and ASCA-, the specificity and sensitivity were largely unchanged at $50 \%$ and $90 \%$, respectively. The PPV improved slightly at 50\%, with a NPV of $90 \%$.

The combination of being pANCA - and ASCA + for CD showed a slightly lower sensitivity of $32 \%$, although the test also held a perfect specificity of $100 \%$. All results regarding the changes in PPV and NPV are shown in Table 2.

Of the five IC patients, four (80\%) were pANCA+ (serology consistent with UC). The fifth patient did not test positive for either pANCA or ASCA IgA or IgG.

\section{DISCUSSION}

The fact that pANCA and ASCA antibodies are associated with $\mathrm{UC}$ and $\mathrm{CD}$, respectively, has been well proven in the literature to date. The question that remains is whether this association is strong enough for these serological markers to be used as primary diagnostic tools for the different subtypes of IBD.

Our results indicate that, for the diagnosis of UC, the pANCA + and ASCA- combination is more accurate than the pANCA test results alone, because the combination test has a $50 \%$ sensitivity and $90 \%$ specificity, with a PPV of $50 \%$. These results are similar to those seen in the literature to date, in which ANCA + titres have been seen in approximately $80 \%$ of UC patients (4). In fact, our results are virtually identical to those of Linskens et al (2), who found a sensitivity and specificity for the pANCA+ and ASCA- combination of $51 \%$ and $94 \%$, respectively.

In contrast to the more accurate results seen with the combination test for the diagnosis of UC, CD identification results did not improve significantly with the combination of tests. In fact, for the pANCA - and ASCA + combination, the sensitivity decreased in comparison to the ASCA+ test, and the specificity and PPV remained unchanged at 100\%. Our results contrast with those found in the literature to date, which show serum ASCA expressed in up to $70 \%$ of CD patients (1). In fact, the majority of the literature to date gives a sensitivity of $44 \%$ to $57 \%$ and a PPV between $75 \%$ and $92 \%$ for the combination test for CD (5).

In general, our results follow the same trends of low sensitivity and high specificity describing these serological markers that have predominated in the literature. In fact, our results are very similar to a recently published meta-analysis that examined the sensitivity and specificity of pANCA and ASCA serology in IBD (6). Where our results deviated from the available literature, however, is in the exceptionally high PPV seen with both the ASCA+ test, as well as with the combination pANCA - and ASCA + test for CD. However, the PPV of these tests is often increased when a study's participants are taken exclusively from an IBD program. In these cases, the referring physician's clinical expertise acts as an effective screening mechanism, and therefore, the subject pool has a reliably high prevalence of IBD (4).

In comparing our results with those in the literature to date, we also need to note that result variability often stems from the use of different cut-off values for a positive titre, differences in the types of assays being used and differences in the types of patients were selected for the study. For example, Zholudev et al (4) found that CD patients with ileal or ileocecal involvement were more likely to have an ASCA+ test. Therefore, a study with a higher number of patients with this CD phenotype is likely to show inflated ASCA+ test results (4).

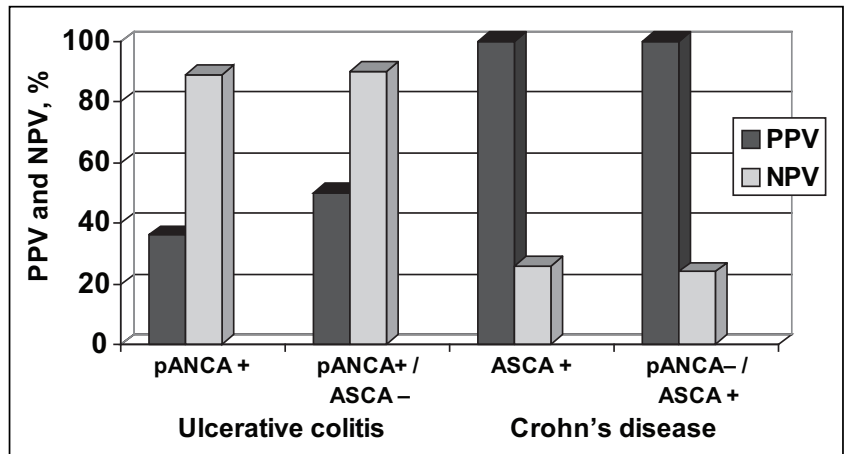

Figure 1) The calculated positive predictive value (PPV) and negative predictive value (NPV) for the serological markers and their combinations. ASCA Anti-Saccharomyces cerevisiae antineutrophil cytoplasmic autoantibody; pANCA Perinuclear antineutrophil cytoplasmic autoantibody; - Negative; + Positive

One should also consider that there are several types of pANCA tests. A pANCA+ test typically reflects antibodies to myeloperoxidase and is seen in association with vasculitis and some autoimmune-type disorders. Nevertheless, other targets can also provide a positive test result with the usual ethanol fixed slides. The pANCA in UC is 'atypical' and is directed against an as yet uncharacterized antigen. It can be distinguished with formalin fixed slides or by using a specific antimyeloperoxidase ELISA.

Clinical relevance for these serological markers may lie in their ability to clarify diagnosis and guide disease management for the subset of IBD patients with IC. According to Linskens et al (2), in approximately $10 \%$ of cases with colonic involvement, an exact diagnosis of the subtype of IBD cannot be made. In our study, four of five of these patients $(80 \%)$ had serology consistent with UC, suggesting that these patients may benefit from an UC-like management plan. In fact, our results are identical to those of both Gupta et al (3) and Olives et al (7), who separately reported that four of every five of their IC patients showed serology consistent with UC.

Most studies comment that the applicability of serological markers for adult populations is far less promising than for the pediatric population because of a complicating factor unique to adults. Reumaux et al (5) further explained this by noting that because the pANCA has recently been observed to be positive in the case of other forms of colitis, as well as in autoimmune hepatitis, it is not useful as a routine screening tool for the adult IBD population. Other studies, such as that by Nakamura et al (1), have demonstrated that the pANCA associated with $\mathrm{UC}$ is, in fact, specific to epitopes within the nucleus, whereas the majority of primary sclerosing cholangitis and autoimmune hepatitis patients show pANCA patterns for cytoplasmic constituents only. Specific applicability remains a controversial point of discussion in the growing debate over the use of serological markers for IBD diagnosis and management.

\section{CONCLUSIONS}

The low sensitivity and high specificity pattern seen in our study suggest that there may be limitations to the applicability of pANCAs and ASCAs for the diagnosis and management of IBD patients. Although our results indicate a statistically more significant PPV for CD than for UC, we suggest that, in both cases, these serological markers may be better used as adjuncts to the clinical workup than as primary 
diagnostic tools. Whether these serological markers may be more useful in family or population screening needs to be addressed with prospective studies. Indeed as the study of these serological markers continues, it is possible that they will prove useful in predicting disease patterns rather than definitively identifying disease itself.

ACKNOWLEDGEMENTS: We would like to thank Carol Johnston for her help with the assays. This work is supported by the Crohn's and Colitis Foundation of Canada, as well as the Cecile Mactaggart Summer Student Research Fund.

\section{REFERENCES}

1. Nakamura RM, Matsutani M, Barry M. Advances in clinical laboratory tests for inflammatory bowel disease. Clin Chim Acta 2003;335:9-20.

2. Linskens RK, Mallant-Hent RC, Groothuismink ZM, et al. Evaluation of serological markers to differentiate between ulcerative colitis and Crohn's disease: pANCA, ASCA and agglutinating antibodies to anaerobic coccoid rods. Eur J Gastroenterol Hepatol 2002;14:1013-8.

3. Gupta SK, Fitzgerald JF, Croffie JM, Pfefferkorn MD, Molleston JP, Corkins MR. Comparison of serological markers of inflammatory bowel disease with clinical diagnosis in children. Inflamm Bowel Dis 2004; $10: 240-4$

4. Zholudev A, Zurakowski D, Young W, Leichtner A, Bousvaros A. Serological testing with ANCA, ASCA, and anti-OmpC in children and young adults with Crohn's disease and ulcerative colitis: Diagnostic value and correlation with disease phenotype. Am J Gastroenterol 2004;99:2235-41.

5. Reumaux D, Sendid B, Poulain D, Duthilleul P, Dewit O, Colombel JF. Serological markers in inflammatory bowel diseases. Best Pract Res Clin Gastroenterol 2003;17:19-35.

6. Reese GE, Constantinides VA, Simillis C, et al. Diagnostic precision of anti-Saccharomyces cerevisiae antibodies and perinuclear antineutrophil cytoplasmic antibodies in inflammatory bowel disease. Am J Gastroenterol 2006;101:2410-22.

7. Olives JP, Breton A, Hugot JP, et al. Antineutrophil cytoplasmic antibodies in children with inflammatory bowel disease: Prevalence and diagnostic value. J Pediatr Gastroenterol Nutr 1997;25:142-8 


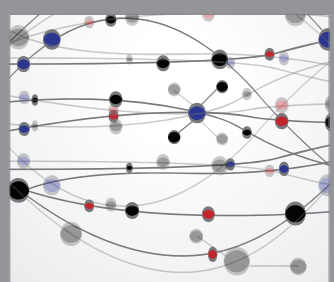

The Scientific World Journal
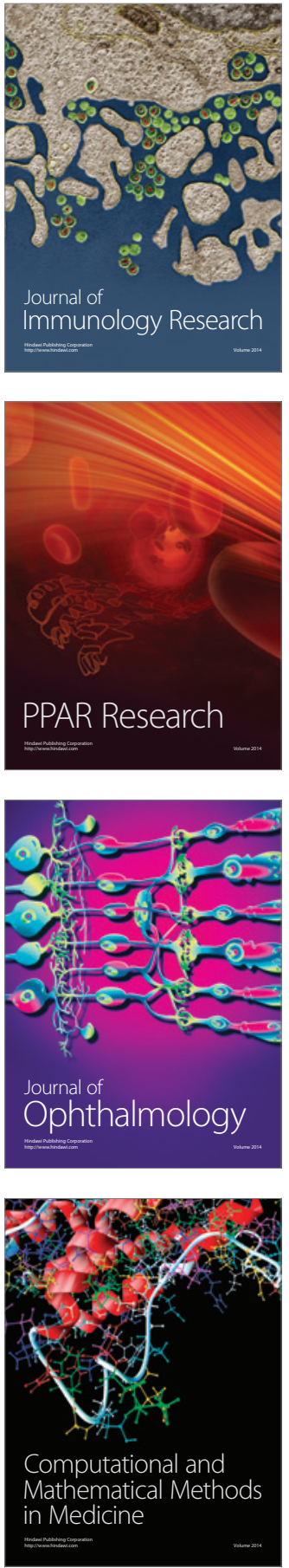

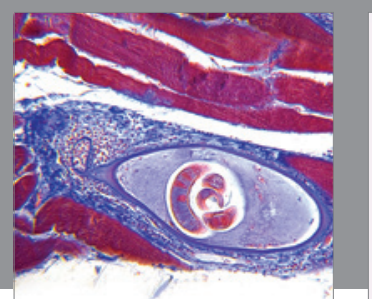

Gastroenterology Research and Practice

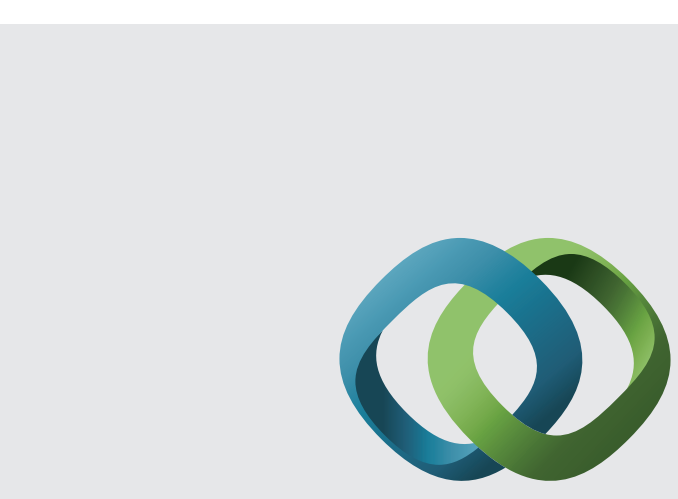

\section{Hindawi}

Submit your manuscripts at

http://www.hindawi.com
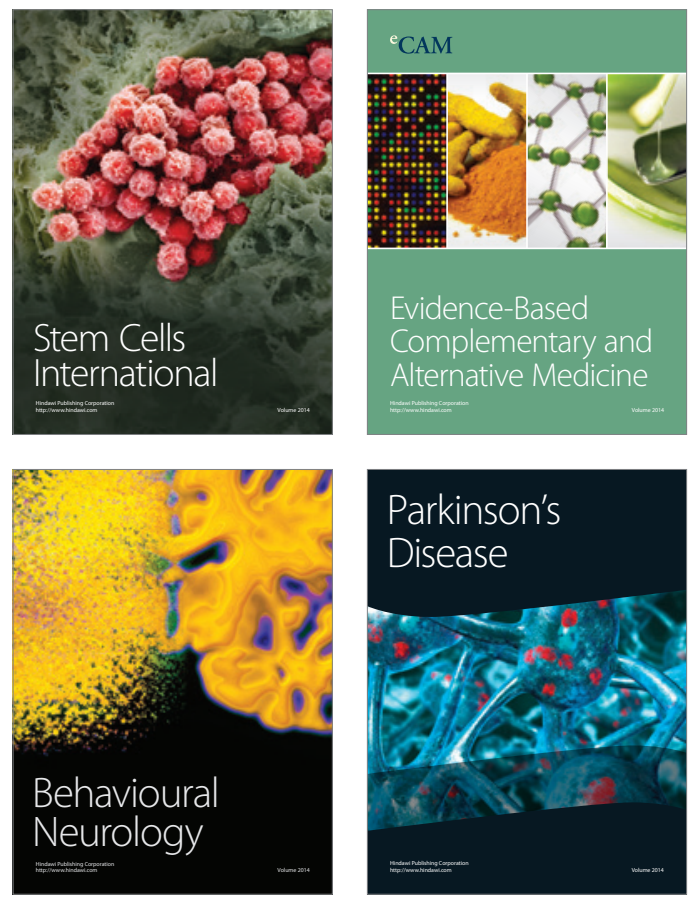
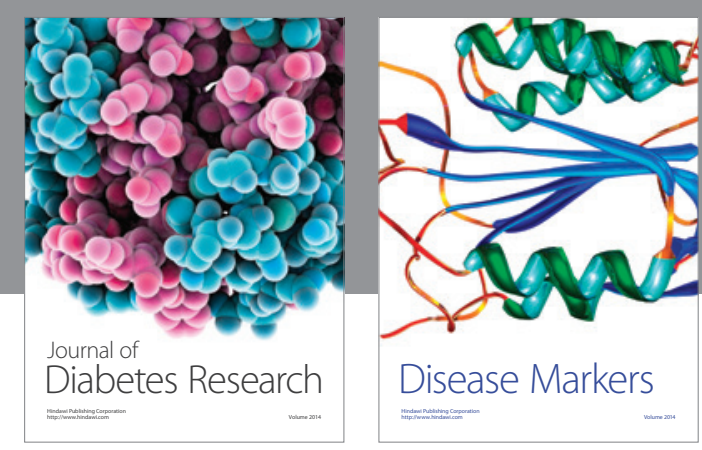

Disease Markers
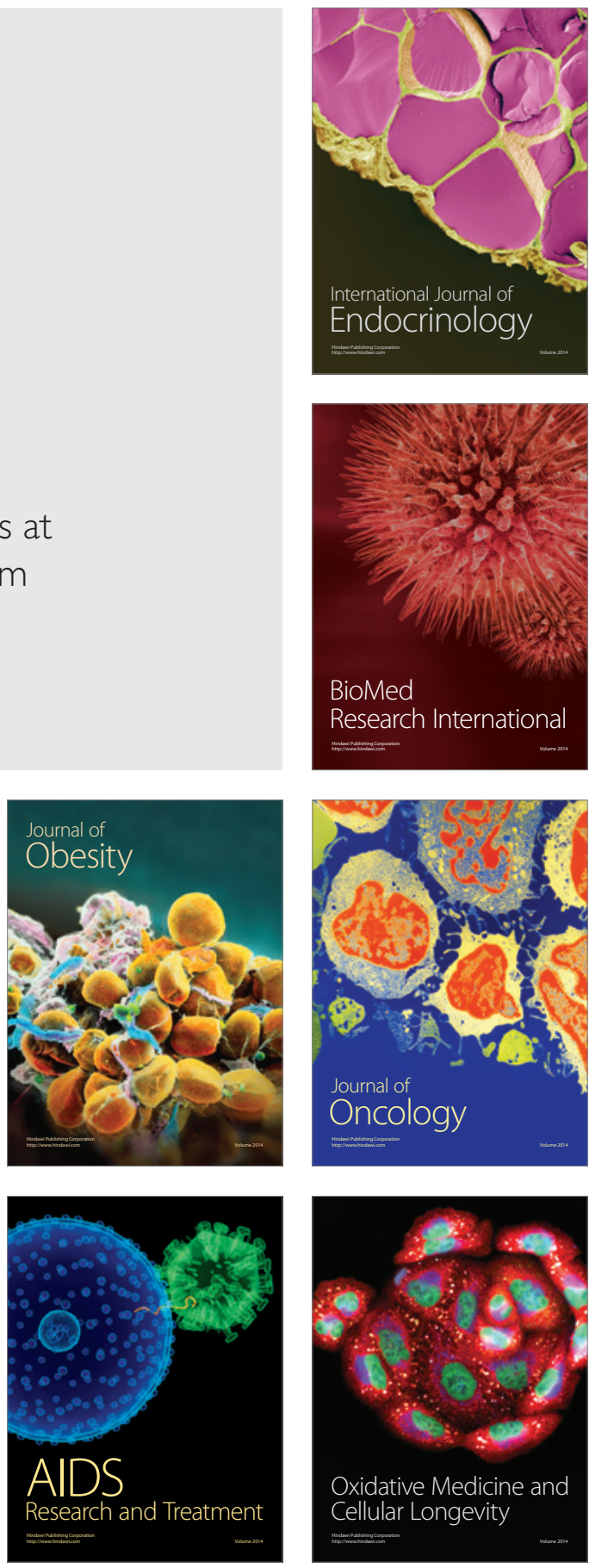